\title{
CORRESPONDENCE Clinical pearls for COVID-19 in children: what do pediatricians need to know?
}

\author{
Pediatric Research (2021) 89:1328-1330; https://doi.org/10.1038/s41390- \\ 020-01123-9
}

In March 2020, the World Health Organization (WHO) declared the 2019 novel coronavirus (severe acute respiratory syndrome coronavirus 2 (SARS-CoV-2)) and its associated disease (coronavirus disease 2019 (COVID-19)) a pandemic. With the rapid spread of COVID-19, there has been gradual recognition of its pathogenesis and clinical characteristics based largely on adult studies. Information on clinical presentation, evaluation and treatment of COVID-19 in children remains limited. We aim to highlight key findings from recent studies to provide clinical pearls for pediatricians caring for children with suspected COVID-19.

\section{CLINICAL PEARL 1: DESPITE RELATIVELY MILD ILLNESS SEVERITY, PROVIDERS MUST BE AWARE OF NOVEL INFLAMMATORY SYNDROMES IN CHILDREN}

A report from the Centers for Disease Control and Prevention (CDC) of 2572 laboratory-confirmed COVID-19 cases suggests that most children in the United States remain asymptomatic or experience mild symptoms. ${ }^{1}$ Children $<18$ years of age accounted for $<2 \%$ of cases. Of these, $6 \%$ required hospitalization and only $2 \%$ required intensive care. The exact reason behind milder illness severity in children is currently unclear.

However, since April 2020, there have been increasing reports of children with SARS-CoV-2 infection presenting with inflammatory features similar to Kawasaki disease. ${ }^{2,3}$ This has recently been termed as multisystem inflammatory syndrome in children (MIS-C). The CDC case definition for MIS-C includes (1) persistent fever $\geq 38.0^{\circ} \mathrm{C}$; (2) laboratory evidence of inflammation; (3) evidence of clinically severe disease requiring hospitalization; (4) multisystem $(\geq 2)$ organ involvement; (5) positive for current or recent SARS-CoV-2 infection; and (6) no alternative plausible diagnoses. ${ }^{2}$ A retrospective study of 58 children in England who met criteria for MIS-C found that all children presented with fever, $31 \%$ with abdominal pain, $52 \%$ with diarrhea, $52 \%$ with an erythematous rash, $45 \%$ with conjunctival injection, $29 \%$ with mucous membrane changes, $26 \%$ with vomiting, $16 \%$ with lymphadenopathy, and $16 \%$ with swollen extremities. ${ }^{4}$ Similar to Kawasaki disease, echocardiogram findings in children with MIS-C include myocardial dysfunction (31\%), coronary artery aneurysms $(15 \%)$, and dilatations (13\%). However, this study also found children with MIS-C to be older than those with Kawasaki disease (mean age of 9 vs 3 years) and to have higher neutrophil count, C-reactive peptide, ferritin, troponin, and $\mathrm{N}$-terminal pro-B-type natriuretic peptide values. This may suggest that this novel syndrome be considered a separate entity from other pediatric inflammatory disorders.

The mechanism underlying MIS-C is unclear. It is currently believed to be an aberrant acquired immune response to SARSCoV-2 with developed antibodies triggering this inflammatory process. ${ }^{4}$ This likely explains why most affected children have positive antibody testing despite a negative PCR test. Children with MIS-C have been treated with intravenous immunoglobulin, aspirin, corticosteroids, and/or immunomodulators. ${ }^{4,5}$ Although most children recover well, pediatricians must remain on high alert as up to one-half of these children may develop shock requiring admission to intensive care units for inotropic support. ${ }^{4,6}$

In addition, an interesting cutaneous manifestation has been described in children with COVID-19. A case series from Spain described 22 pediatric patients presenting with acral erythematoviolaceous purpuric lesions consistent with chilblains or pernio. ${ }^{7}$ These lesions were all located on the toes and lateral aspects of the feet and have been popularly dubbed "COVID toes" in the media. The lesions are typically non-tender and non-pruritic. To a lesser extent, they can also be located on the periumbilical areas of the fingers. Most of these lesions spontaneously resolve 3-5 weeks after onset.

\section{CLINICAL PEARL 2: CLINICAL PRESENTATION, LABORATORY INVESTIGATIONS, AND RADIOLOGIC FINDINGS IN CHILDREN DIFFER FROM ADULTS}

A US report of 291 children with confirmed COVID-19 found that the most common symptoms were fever (56\%) and cough $(54 \%){ }^{1}$ Nearly one-third of children had gastrointestinal complaints of abdominal pain, nausea/vomiting, and diarrhea. Other nonspecific symptoms included rhinorrhea, sore throat, headache, and myalgia. These figures vary greatly from adults in the same report, in whom fever and cough were reported in $71 \%$ and $80 \%$ of patients, respectively.

Laboratory investigations of 20 infected children from the Wuhan Children's Hospital found $90 \%$ of children having a normal or low white blood cell count, which may be increased in adult patients. ${ }^{8}$ Other pediatric studies report normal inflammatory markers including C-reactive protein, procalcitonin, and ferritin. ${ }^{9}$ These indicate a lack of immune dysregulation that is more commonly seen in adults and may provide another explanation for milder disease severity in children. An exception is the MIS-C presentation likely due to a dysregulated inflammatory response, which is characterized by significantly elevated inflammatory markers. ${ }^{4}$

In cases of severe disease in children necessitating hospital admission, computed tomography (CT) of the chest may be useful to provide more information. The most common CT findings in the pediatric population include unilateral and bilateral subpleural ground-glass opacities and consolidation with a surrounding halo sign. ${ }^{8}$ The halo sign is not routinely seen in CT images from adult patients and is suggested to be a "characteristic finding" in affected children. There has been more new interest in the use of lung ultrasound given its benefits of being radiation-free and portable, which would limit patient transport within the hospital and minimize exposure to other patients and staff. ${ }^{10}$ Lung ultrasound findings in children include subpleural consolidations and interstitial B lines. Further, improvements in these parameters were seen on repeat ultrasound prior to discharge, which may suggest its utility in real-time assessment of recovering patients. 
CLINICAL PEARL 3: NEONATES ARE AT A GREATER RISK OF SEVERE ILLNESS AND REQUIRE SPECIAL CONSIDERATIONS Although children overall are less affected by COVID-19, very young infants are more vulnerable to the disease. The largest retrospective study of 731 Chinese children with confirmed COVID-19 found that $54 \%$ of all children with critical severity of illness were $<1$ year of age. ${ }^{11}$ In the US, neonates account for $15 \%$ of all pediatric cases. ${ }^{1}$ Therefore, special attention should be given to those in the neonatal setting. Clinicians caring for neonates should be aware that there is currently no evidence to suggest vertical transmission or prenatal infection of COVID-19 from infected mothers to neonates. ${ }^{12}$ The transmission of SARS-CoV-2 to neonates is thought to be postnatal from infected mothers and other close contacts. ${ }^{13}$ Clinical signs include fever, lethargy, increased work of breathing, vomiting, diarrhea, and feeding intolerance. Preterm infants infected with SARS-CoV-2 have also been reported in whom COVID-19 manifestations are difficult to distinguish from prematurity-related respiratory comorbidities. ${ }^{14}$ The CDC currently recommends that all infants, including those who are asymptomatic, born to mothers with confirmed or suspected COVID-19 be tested at approximately $24 \mathrm{~h}$ of age. ${ }^{13}$ Pending the result, these infants should be presumed infectious and remain isolated from other neonates. The CDC does not prohibit infected mothers from breastfeeding their newborns as long as mothers follow appropriate respiratory etiquette. Wellappearing neonates can be carefully discharged while awaiting results of the SARS-CoV-2 testing.

\section{CLINICAL PEARL 4: WITH THE REOPENING OF PUBLIC AREAS AND SCHOOLS, PROVIDERS MUST CONTINUE TO FOCUS ON KEEPING CHILDREN HEALTHY AND RESILIENT}

As in adults, the COVID-19 outbreak and its lockdown has been extremely disruptive to children's daily routines. There is some evidence that school closure for children may be minimally impactful in reducing spread of COVID-19 and may in fact be harmful. ${ }^{15}$ The stay-at-home orders have led to reduced access to social support services for children and their families. ${ }^{16}$ These include children who socialize with their friends and peers at school as coping mechanisms for everyday problems and those children with special education needs who rely on close therapist contact to develop essential skills. ${ }^{17}$ Conversely, parents have been tasked with additional responsibilities, including facilitating online learning that can stress families as a whole. As restrictions on school closures and community services begin to loosen, pediatricians must continue to work with families to ensure children emerge healthy and resilient. Strategies include encouraging families to plan daily schedules to limit children's screen time and incorporate regular physical activity and exercise. ${ }^{18}$ Further, physical distancing rather than social distancing should be promoted to allow children and their families to remain socially connected with loved ones. Parents should also be educated on how their child may display signs of stress including irregular sleeping habits, dietary habits, difficulty with attention and concentration, and excessive worrying or sadness. Encourage parents to talk honestly but positively about the pandemic in ways that their child can understand and to seek help when needed.

A recent study from China found that $96 \%$ of children with COVID-19 were household contacts of adults who displayed symptoms of the infection earlier. ${ }^{19}$ Hence, the most common exposure history in children to the SARS-CoV-2 virus is through family clustering. Therefore, pediatricians must remain on high alert for children in households where family members have been diagnosed with COVID-19 even though the child may be initially asymptomatic. Conversely, it was originally thought that infected children who appear asymptomatic may play a significant role propagating community spread. However, evidence does not lean toward children being a major vector for disease spread. ${ }^{20}$ This provides some reassurance that school-based transmission could be managed in the upcoming months as schools reopen. To further lessen the risk of transmission with the reopening of schools, hygiene etiquette with frequent handwashing must remain a priority for families and care providers. Lastly, $40-50 \%$ of all children with COVID-19 can be co-infected with other respiratory pathogens. ${ }^{8,19}$ Heading into the "flu season" later this year, it will be important for pediatricians to consider SARS-CoV-2 co-infection in children with more common pathogens, such as the respiratory syncytial virus.

\section{ACKNOWLEDGEMENTS}

We thank Dr. Mark Anselmo, a pediatric respirologist at the Alberta Children's Hospital in Calgary, Alberta, Canada, for proofreading this article and providing helpful suggestions.

\section{AUTHOR CONTRIBUTIONS}

R.V. performed the literature search, drafted the initial manuscript, and reviewed and revised the manuscript. R.A. conceptualized and designed the project, drafted the initial manuscript, and reviewed and revised the manuscript. Both authors approved the final manuscript as submitted and agree to be accountable for all aspects of the work.

\section{ADDITIONAL INFORMATION}

Competing interests: The authors declare no competing interests.

Patient consent: Patient consent was not required for this study.

Publisher's note Springer Nature remains neutral with regard to jurisdictional claims in published maps and institutional affiliations.

Rahul Verma ${ }^{1}$ and Reshma Amin ${ }^{2,3}$

${ }^{1}$ Department of Pediatrics, Children's Hospital, Western University, London, ON, Canada; ${ }^{2}$ Division of Respiratory Medicine, Department of Pediatrics, Hospital for Sick Children, University of Toronto, Toronto, ON, Canada and ${ }^{3}$ Child Health Evaluative Sciences, SickKids Research Institute, Toronto, ON, Canada Correspondence: Reshma Amin (Reshma.Amin@sickkids.ca)

\section{REFERENCES}

1. Centers for Disease Control and Prevention (CDC). Coronavirus disease 2019 in children - United States, February 12-April 2, 2020. MMWR Morb. Mortal Wkly. Rep. 69, 422-426 (2020)

2. Centers for Disease Control and Prevention (CDC). Multisystem inflammatory syndrome in children (MIS-C) associated with coronavirus disease 2019 (COVID19). https://emergency.cdc.gov/han/2020/han00432.asp (2020).

3. Centers for Disease Control and Prevention (CDC). Information for pediatric healthcare providers. https://www.cdc.gov/coronavirus/2019-ncov/hcp/pediatrichcp.html (2020).

4. Whittaker, E. et al. Clinical characteristics of 58 children with a pediatric inflammatory multisystem syndrome temporally associated with SARS-CoV-2. JAMA 324, 259-269 (2020).

5. Belhadjer, Z. et al. Acute heart failure in multisystem inflammatory syndrome in children (MIS-C) in the context of global SARS-CoV-2 pandemic. Circulation. 142, 429-436 (2020).

6. Children's Hospital of Philadelphia. Emergency department, ICU and inpatient clinical pathway for evaluation of possible multisystem inflammatory syndrome (MIS-C). https://www.chop.edu/clinical-pathway/multisystem-inflammatory-syndrome-mis-cclinical-pathway (2020).

7. Andina, D. et al. Chilblains in children in the setting of COVID-19 pandemic. Pediatr. Dermatol. https://doi.org/10.1111/pde.14215 (2020).

8. Xia, W. et al. Clinical and CT features in pediatric patients with COVID-19 infection: different points from adults. Pediatr. Pulmonol. 55, 1169-1174 (2020).

9. Tan, Y.-p et al. Epidemiologic and clinical characteristics of 10 children with coronavirus disease 2019 in Changsha, China. J. Clin. Virol. 127, 104353 (2020).

10. Denina, M. et al. Lung ultrasound in children with COVID-19. Pediatrics 146, e20201157 (2020). 
11. Dong, Y., et al. Epidemiological characteristics of 2143 pediatric patients with 2019 coronavirus disease in China. Pediatrics 145, e20200702 (2020).

12. Chen, $H$. et al. Clinical characteristics and intrauterine vertical transmission potential of COVID-19 infection in nine pregnant women: a retrospective review of medical records. Lancet 395, 809-815 (2020).

13. Centers for Disease Control and Prevention (CDC). Evaluation and management considerations for neonates at risk for COVID-19. https://www.cdc.gov/ coronavirus/2019-ncov/hcp/caring-for-newborns.html (2020).

14. Piersigilli, F. et al. COVID-19 in a 26-week preterm neonate. Lancet Child Adolesc. Health 4, 476-478 (2020).

15. Viner, R. M. et al. School closure and management practices during coronavirus outbreaks including COVID-19: a rapid systematic review. Lancet Child Adolesc. Health 4, 397-404 (2020).
16. Stark, A. M., White, A. E., Rotter, N. S. \& Basu, A. Shifting from survival to supporting resilience in children and families in the COVID-19 pandemic: lessons for informing U.S. mental health priorities. Psychol. Trauma 12, S133-S135 (2020).

17. Lee, J. Mental health effects of school closures during COVID-19. Lancet Child Adolesc. Health 4, 421 (2020).

18. Centers for Disease Control and Prevention (CDC). Keep children healthy during the COVID-19 outbreak. https://www.cdc.gov/coronavirus/2019-ncov/daily-lifecoping/children.html (2020).

19. Wu, Q. et al. Coinfection and other clinical characteristics of COVID-19 in children. Pediatrics 146, e20200961 (2020)

20. Davies, N. G. et al. Age-dependent effects in the transmission and control of COVID-19 epidemics. Nat. Med. 26, 1205-1211 (2020). 